\title{
Comparison of holographic setups used in heat and mass transfer measurement
}

\author{
R. Doleček ${ }^{1,2, a}$, P. Psota ${ }^{1,2}$, V. Léd1 ${ }^{1,2}$, T. Vít ${ }^{1,2}$ and V. Kopecký ${ }^{1}$ \\ ${ }^{1}$ Technical University of Liberec, Studentská 2, 46117 Liberec, Czech Republic \\ ${ }^{2}$ IPP CAS (TOPTEC), Sobotecká 1550, Turnov, Czech Republic
}

\begin{abstract}
The authors of the paper deal with measurement of heat and mass transfer for several years and they have frequently used few techniqes for measurement of refractive index distribution based on holographic interferometry. Some of the well known techniques have been modified some and some new ones developped. Every technique could be applied with success in different type of meassurement and obviously every one has set of properties making them unique. We decided to digest few different basic techniques and describe its properties in this paper with the aim to help the reader select the proper one for their measurement. The list of techniques and its properties is not comprehensive but schould serve as a basic orientation in the field.
\end{abstract}

\section{Introduction}

Heat and mass transfer measurement is very important discipline in thermomechanics. Many different techniques have been applied since its systematic measurement started. The well known and most applied is probably the Schlieren technique which recently notched up small renaissance when colour scale and fast cameras have been brought in. Schlieren technique [1] is in use for almost 150 years now and still is a valuable tool in brief previews of physical problems mostly because it is simple and illustrative on the other hand quantitative information gain is always rather limited due to many factors like observed change integration over the ray trajectory or sensitivity of the setup in dependence of $\mathrm{F}$ number of the optical system. Holographic interferometry $\mathrm{HI}$ and especially its recent digital form DHI opened new perspectives in heat and mass transfer measurement. HI already occupies its important place in this field for almost 40 years but techniques like tomography or averaging of hundreds of frames were made feasible after "digital age" arrival. We adapted some classical techniques to digital form and developed some new techniques from scratch like synchronizing in coherent phenomena measurement. Techniques which were adopted or developed and took its place in our standard measurement procedures are subject of this paper. We still continue in development of new enhanced techniques. Measurement of small pressure changes and development in the air is now taken as a major discipline which we are willing to progress in.
Those small changes are translated to very tiny refractive index change so we aim mainly to sensitivity increase progress.

\section{DHI principles}

DH employs CCD sensor for recording of the interference pattern formed by a reference wave $U_{r}$ and an object wave $U_{o}$ transmitting through the area of interest (temperature field). The recorded pattern is called the hologram $h$ and is expressed by relation

$$
\left|U_{o}+U_{r}\right|^{2}=\left|U_{o}\right|^{2}+\left|U_{r}\right|^{2}+U_{o} U_{r}^{*}+U_{r} U_{o}^{*}
$$

The maximum angle $\theta_{\max }$ between these two waves is related to spatial frequency, which the given CCD can record without violating the Nyquist criterion, (it means two pixels for one interference fringe). This condition is expressed by the following relation

$$
\theta_{\max }=2 \arcsin \left(\frac{\lambda}{4 \Delta \xi}\right) \approx \frac{\lambda}{2 \Delta \xi},
$$

where $\Delta \xi \times \Delta \xi$ is the pixel size.

With comparison with classical $\mathrm{HI}$ is the reconstruction process done completely numerically. Let's assume that the temperature field is located at a distance $d$ far from the CCD. The reconstruction is calculated by multiplication of the digital hologram $h$ with the complex amplitude of the reference wave ${ }^{*}$. The reconstructed complex wave field $U(n, m)$ in the image plane is calculated by the Sommerfeld formula, which

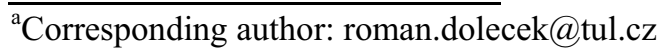


describes the diffraction of a light wave at the hologram in distance $d$ far from the gologram. The Fresnel approximation of the Sommerfeld integral is given [2]

$$
\begin{aligned}
& U(n \Delta x, m \Delta y)=e^{j \pi \lambda d\left(\left(\frac{n \Delta x}{N \Delta \xi}\right)^{2}+\left(\frac{m \Delta y}{M \Delta \eta}\right)^{2}\right)} \times \\
& \times \sum_{k=1}^{N} \sum_{l=1}^{M} h(k \Delta \xi, l \Delta \eta) r^{*}(k \Delta \xi, l \Delta \eta) e^{\frac{j \pi}{\lambda d}\left((k \Delta \xi)^{2}+(l \Delta \eta)^{2}\right)} e^{-j 2 \pi\left(\frac{k n}{N}+\frac{l m}{M}\right)}
\end{aligned}
$$

Where $j=\sqrt{-1}$ and $\lambda$ is wavelength of the used light. The count of pixel in perpendicular direction describes $N \times M$ and pixel center-to-center distance of used CCD is $\Delta \xi \times \Delta \eta$.

In practical applications the pixel numbers $N, M$ and the pixel size $\Delta \xi \times \Delta \eta$ are defined by the default propertyof digital camera. Image plane distances $\Delta x, \Delta y$ are given by the size of a frame, where the reconstructed object wave is displayed and depend on distance $d$ and wavelength $\lambda$ :

$$
\Delta x=\frac{\lambda d}{N \Delta \xi} \text { and } \quad \Delta y=\frac{\lambda d}{M \Delta \eta} .
$$

Reconstructed intensity I is proportional to $I \approx|U|^{2}$.

The Fresnel transformation can be effectively calculated with use of FFT algorithm (Fast Fourier Transform). If we take a look at the last term of the formula (3) one can recognize the Fourier transform definition. Therefore, the formula (3) becomes [2]:

$$
\begin{aligned}
& U(n \Delta x, m \Delta y)=\frac{j}{d \lambda} \exp \left(-\frac{j \pi}{\lambda d}\left[(n \Delta x)^{2}+(m \Delta y)^{2}\right]\right) \times \\
& \times \mathcal{F}^{-1}\left\{f ( k \Delta \xi , l \Delta \eta ) U r ^ { * } ( k \Delta \xi , l \Delta \eta ) \operatorname { e x p } \left(-\frac{j \pi}{\lambda d}\left[(k \Delta \xi)^{2}\right.\right.\right. \\
& \left.\left.\left.\quad+(l \Delta \eta)^{2}\right]\right)\right\},
\end{aligned}
$$

where $\mathcal{F}^{-1}$ denotes the inverse Fourier transform and the last term in (4) is called chirp function, which mathematically corresponds to impulse response of linear system.

In the DHI at least two digital holograms $h_{1}, h_{2}$ are captured. The first hologram corresponds toan initial (reference) state of temperature field and the second hologram is captured when the temperature field has been varied. The phase distributions of the both reconstructed fields are obtained by

$$
\begin{aligned}
& \varphi_{1}(n, m)=\arctan \frac{\operatorname{Im}\left\{U_{1}(n, m)\right\}}{\operatorname{Re}\left\{U_{1}(n, m)\right\}}, \\
& \varphi_{2}(n, m)=\arctan \frac{\operatorname{Im}\left\{U_{2}(n, m)\right\}}{\operatorname{Re}\left\{U_{2}(n, m)\right\}}
\end{aligned}
$$

The interference phase $\Delta \varphi(n, m)$ relates to the temperature field variation and can be determined in a point wise manner by a modulo $2 \pi$ subtraction

$$
\Delta \varphi=\left\{\begin{array}{lll}
\varphi_{1}-\varphi_{2} & \text { if } & \varphi_{1} \geq \varphi_{2} \\
\varphi_{1}-\varphi_{2}+2 \pi & \text { if } & \varphi_{1}<\varphi_{2}
\end{array}\right.
$$

Let's assume the first hologram is recorded with refractive index $n_{1}$ and the second hologram with $n_{2}$. The relation between refractive index change $\Delta n=n_{2}-$ $n_{1}$ and interference phase is given

$$
\Delta \varphi=\frac{2 \pi}{\lambda} \oint_{L}\left(\Delta n-n_{\infty}\right) d l
$$

Where $d l$ denotes the differential distance along the line $L, n_{\infty}$ is refractive index of surrounding air. From the general physically mathematical point of view one can consider the temperature field varying in only one direction to be the easiest case. Since there is no refractive index variation along the object length $\mathrm{L}$, formula for the interference phase calculation (8) becomes:

$$
\Delta \varphi=\frac{2 \pi}{\lambda} L\left(\Delta n-n_{\infty}\right)
$$

In axis-symmetrical temperature fields, the refractive index (or temperature distribution) is a function of radius $\mathrm{r}$ and formula (8) becomes:

$$
\Delta \varphi=\frac{4 \pi}{\lambda} \int_{x}^{\infty} \frac{r\left(\Delta n-n_{\infty}\right)}{\sqrt{r^{2}-x^{2}}} d r
$$

For the reconstruction of circular symmetric temperature field one can use inversion of Abel transform corresponding to the right-hand side of Eq. (10).

The determination of asymmetric fields requires the analysis of a large number of holographic interferograms by methods of tomography. In this case the line integral transform defined in (6) is mathematically equivalent to Radon Transform [3]:

$$
g(s, \theta)=\iint_{-\infty}^{+\infty} f(x, y) \delta(x \cos \theta+y \sin \theta-s) d x d y,
$$

where $f(x, y)$ is the refractive index distribution in Cartesian coordinates, $\delta$ is the Dirac pulse, $\theta$ is the angle of projection and $s$ is the shift distance from the center. The data obtained by Radon Transform $g(s, \theta)$ is called sinogram, because the Radon transform of a Dirac delta function is a distribution supported on the graph of a sine wave. In practice, the sinogram is a $2 \mathrm{D}$ array containing the projections from different angles in columns of the array. Reconstruction is based on back-projection mathematically described by Inverse Radon Transform $[4,5]$ :

$$
f(x, y)=\int_{0}^{\pi} g(x \cos \theta+y \sin \theta, \theta) d \theta
$$

The back-projection operation propagates the measured sinogram back into the image space along the projection paths. Further, images for all rows are stacked to get 3D volume data.

The temperature and refractive index relation is given by Gladston-Dale equation

$$
n-1=K . \rho \text {. }
$$

Where $K$ is Gladston-Dale constant which is property of gas. The Gladston-Dale constant is almost independent on pressure or temperature under the moderate physical conditions. It is slightly dependent on wavelength of the light. The density $\rho$ of the gas can be calculated from the pressure $P$, the molecular weight $M$ and absolute temperature $T$ via the ideal gas equation.

$$
\rho=\frac{M P}{R T}
$$

Combined with the universal gas constant, $R=8,3143 \mathrm{JK}^{-1} \mathrm{~mol}^{-1}$. This with (13) yields

$$
n-1=\frac{K M P}{R T}
$$




\section{Single pass $\mathrm{DHI}$ setup}

The Mach-Zehnder type of interferometer is the most used setup for the measurement of transparent objects in $\mathrm{HI}$ as well as in DHI. Figure 1 shows its principle schema [6]. Laser beam emitted from laser head is divided by polarizing beam splitter $\mathrm{BS}_{1}$ equipped with half wave plates. Half-wave plates are used to adjust intensity and polarization of beams in each arm of interferometer. Both beams are further filtered by spatial filters SF and collimated. Collimated beam no. 2 is directed to a nonpolarizing beam splitterBS ${ }_{2}$. One part of light-wave passes directly through the $\mathrm{BS}_{2}$ and reaches screen $\mathrm{S}$ where the beam is absorbed. Second part is reflected in the $\mathrm{BS}_{2}$ to objective $\mathrm{O}$ and strikes $\mathrm{CCD}$ chip of the camera. Collimated beam no. 2 goes through measured phase-object and is reflected by mirror $\mathrm{M}_{2}$ to beamsplitterBS $\mathrm{B}_{2}$. Again, one part of this beam part hits the screen $\mathrm{S}$ and second one reaches objective $\mathrm{O}$ and CCD chip. Both waves that are impinging on CCD chip interfere there. Two digital holograms capturing phaseobject in two different physical states are recorded and further numerically processed by method described in previous chapter. The area of interest is limited by the size of the beamsplitter $\mathrm{BS}_{2}$ in our case $50 \times 50 \mathrm{~mm}$

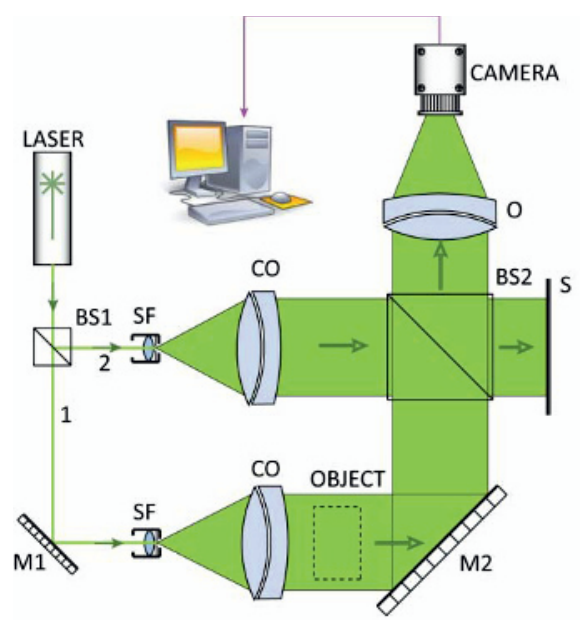

Fig.1: Experimental holographic setup schematics of MachZehnder type of interferometer. BS $S_{1}$-polarizing beam splitter, $\mathrm{SF}$-spatial filter, CO-colimation objective, $\mathrm{BS}_{2}$-nonpolarizing bemasplitter, O-objective lens, M-mirror, S-screen.

Figure 2 shows the reconstructed interference phase distribution and corresponded temperature field of the resistor and resistive spiral heated by the electric current. Fields are the projections of the temperature changes in direction of the object beam.

The main advantage of this interferometric setup is its relative simplicity. The adjustment of the single component requires an exercise and at least certain handiness. On the other hand the sensitivity of the interferometer can be insufficient for low temperature changes.

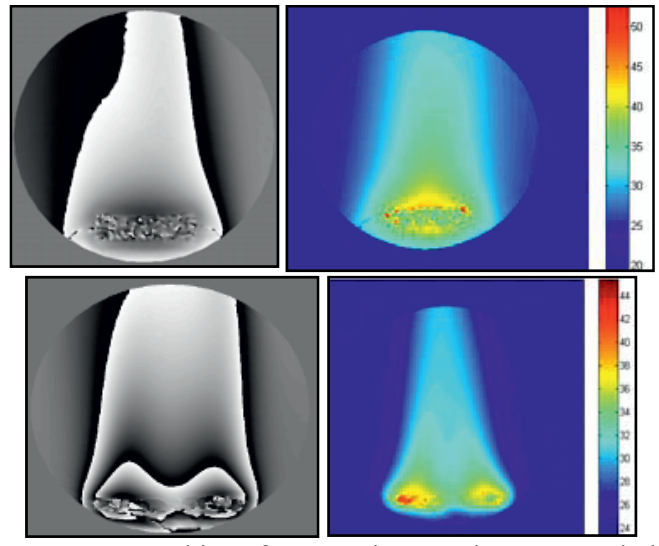

Fig.2: Reconstructed interference phase and corresponded temperature field measured on Mach-Zehnder interferometer.
pros + Relatively simple to built. Low complexity. Good for the phenomena where strong phase change may occur in small area (low gradients).
cons - Small sensitivity.

\section{Double path DHI setup}

For the measurement of small phase changes is the sensitivity of Mach-Zehnder type of interferometer insufficient. Altered setup based on Michelson interferometer offers two-times higher sensitivity compared to Mach-Zehnder interferometer. Working principle of such interferometer is shown in Fig. 3. The laser beam is divided by polarizing beam splitter $\mathrm{BS}_{1}$ and two new originated beams are modified similarly as it was in the previous setup. Beam no. 1 is reflected by mirror $\mathrm{M}_{2}$ to divider $\mathrm{BS}_{2}$. One part of the beam no. 1 goes directly through divider and objective $\mathrm{O}$ to $\mathrm{CCD}$ chip. Second part is reflected to collimating objective CO. Collimated beam no. 2 enters the beam splitter $\mathrm{BS}_{2}$. One part of the beam no.2 is reflected in direction to $\mathrm{M}_{2}$, second part passes through the measured object. Mirror $\mathrm{M}_{3}$ is placed behind the object and it reflects the beam back through the object to the beam splitter ND. There the beam undergoes second division and part of it is directed through the objective $\mathrm{O}$ to the CCD chip.

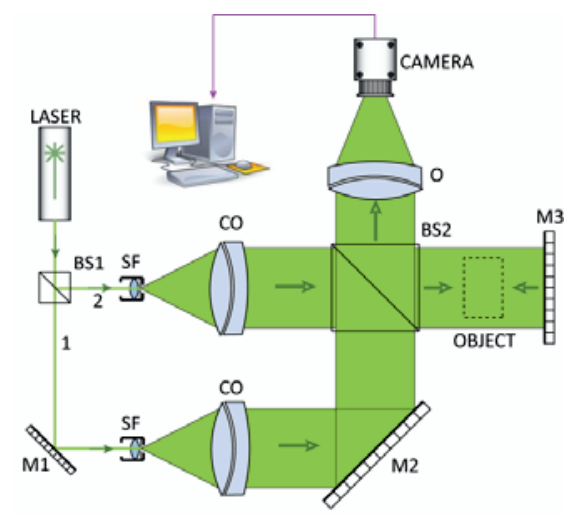

Fig. 3:Experimental holographic setup schematics of Michalson type of interferometer with double pass. $B S_{1}$-polarizing beam splitter, SF-spatial filter, CO-colimation objective, $B S_{2^{-}}$ nonpolarizing bemasplitter, O-objective lens, M-mirror. 
This setup allows to the beam to pass through the measured object twice which results in higher sensitivity. It can be seen in double fringe-count in reconstructed interference phase (see Fig. 4). On the other hand such setup is more complicated and difficult to adjust to correct performance. The object should be placed as close to the mirror $\mathrm{M}_{3}$ as possible in order to be guaranteed the some way through the object for both beams. There is also higher energy loss on the divider $\mathrm{BS}_{2}$. Therefore usage this setup requires longer capture time for the hologram capture in comparison with Mach-Zehnder type of interferometer. In the measurement of the fast varying temperature fieldsthe long capture time can causelow contrast or the smudging of the hologram. There are several possible solutions depending on the problem under investigation. One can use higher power laser or more sensitive high speed camera or the pulse laser with synchronized camera.

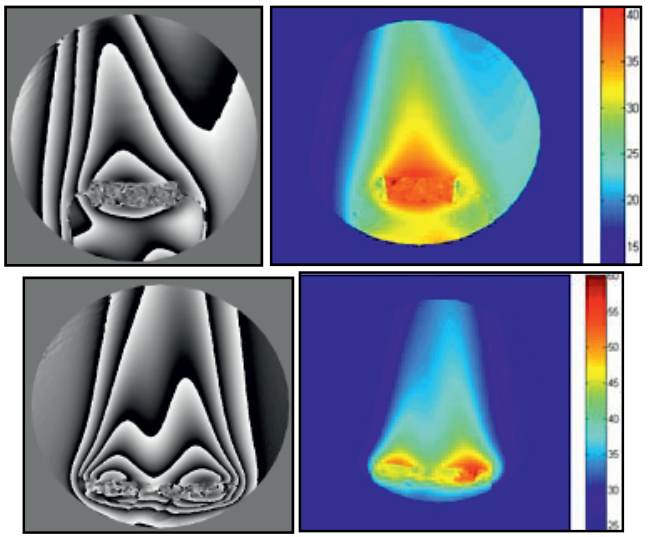

Fig.4: Reconstructed interference phase and corresponded temperature field measured on Michalson type of interferometer.

\section{pros + Double the sensitivity than the standard MZ setup. \\ Moderate complexity, \\ Due to higher sensitivity - possibility to measure in media like He. \\ cons - Moderate difficulty to adjust. Becomes costly.}

\subsection{Coherent phenomena measurement}

For measurement of the fast periodical temperature field development is not necessary to use always the high speed camera. One can consider this field to coherent phenomenon and use synchronization of the fieldgenerator-driverwith the camera capture time. The external trigger of the camera enables one to capture the hologram with a precisely defined delay. Since the synchronization frequency is higher than the camera frame rate, stroboscopic values must be applied.The hologram is not captured after each synchronization signal but some periods of the phenomenon are skipped (see Fig. 5). Therefore it is possible to measure very high frequency phenomena with use of a lower frame rate camera [7].

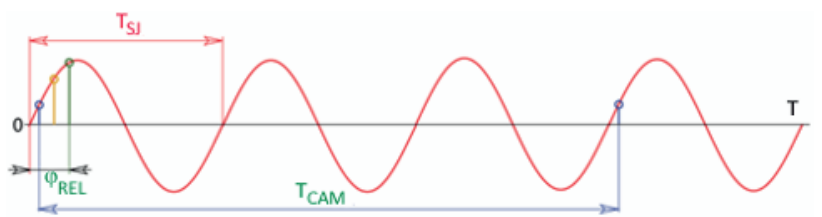

Fig. 5: Synchronization and triggering between the camera and phenomenon driver. $\mathrm{T}_{\mathrm{SJ}}$ - period of the phenomenon, $\varphi_{\mathrm{REL}}-$ relative phase of the phenomenon (defined delay), $\mathrm{T}_{\mathrm{CAM}}-$ capture time of the camera

For demonstration we selected the synthetic jet SJ [8]. SJ are jets of fluid that are generated by pushing or pulling a fluid through an orifice by the interactions within the train of counter-rotating vortex pairs. SJ are generated with frequency $15 \mathrm{~Hz}$ by one pairs of blowers. Though we used double sensitivity interferometer the temperature changes were too low. Therefore we placed a heating cartridge in the space in between the blowers that heats up the air of the flux of air flowing from the orifice. The heating cartridge was controlled by a connected PID regulator. The principle schema is showed in Fig. 6.

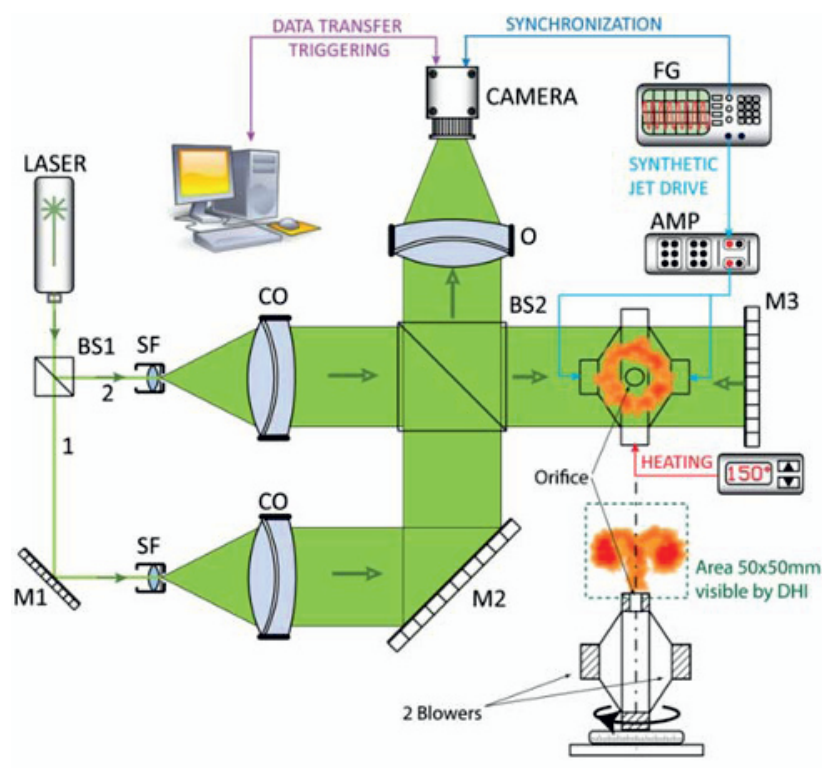

Fig. 6: Principle schema of the interferometer with synchronization of the camera and driver of temperature field. $\mathrm{FG}$ - functions generator, AMP - amplifier.

In Fig. 7 the consequence of the triggering process of a coherent $\mathrm{SJ}$ phenomenon and the digital camera is shown. It is proofed the coherence of the SJ phenomenon. Shown reconstructed fields correspond to the same relative time but in different periods of the repeated phenomenon. The down row images are standard deviation of each pixel divided by its own value in a percentage scale. It can be seen the differences between the fields are less than $5 \%$. This uncertainty is caused by the random fluctuation. However it can be reduced by an averaging of all fields correspond to the same relative time. The temperature field dynamic evolution in time is shown in Fig. 8. The holograms were captured at different time delays $-0 \mathrm{~ms}$, $8 \mathrm{~ms}$ and $16 \mathrm{~ms}$. 


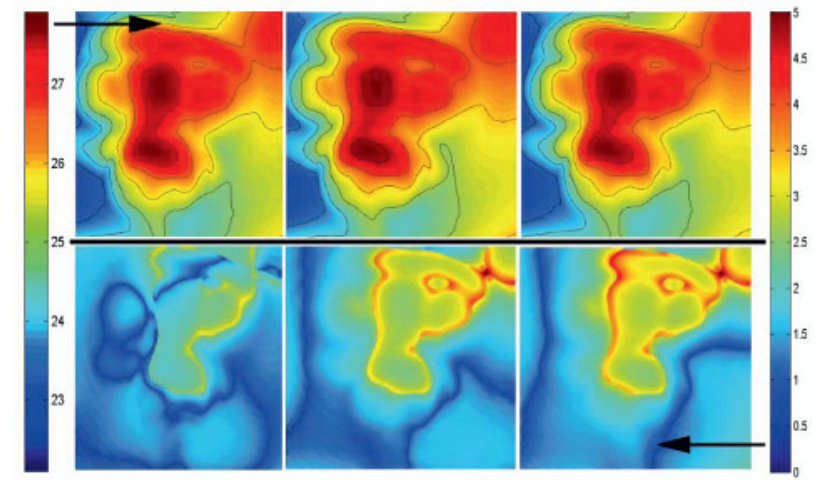

Fig.7: The consequence of a precise camera and SJ driver synchronization. First row shows temperature field $\left[{ }^{\circ} \mathrm{C}\right]$ captured at the same time delay $16 \mathrm{~ms}$ in different cycles of the periodical phenomenon. The bottom row displays the standard deviations [\%] of temperature field distributions.

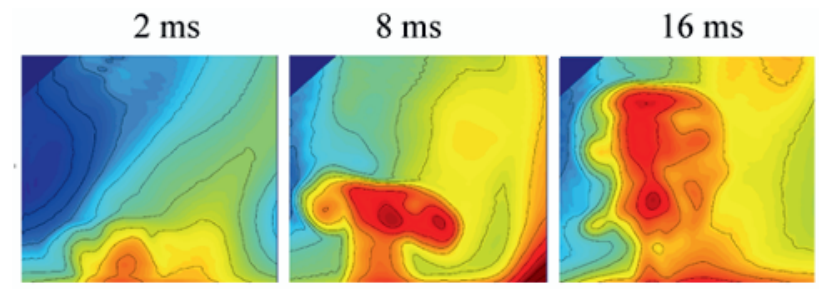

Fig. 8: The dynamic evaluation of temperature field $\left[{ }^{\circ} \mathrm{C}\right]$.

$\begin{aligned} \text { pros }+ & \text { Double the sensitivity than the standard MZ } \\ & \text { setup. } \\ & \text { Fast periodic (coherent) phenomena } \\ & \text { measurement. } \\ & \text { Possibility to average many frames for noise } \\ & \text { suppression. } \\ \text { cons - } & \text { Rather complex to built up an and adjust. } \\ & \text { Costly due to need of camera with sync. }\end{aligned}$

\subsection{Tomography based reconstruction}

In the easiest case temperature fields are two-dimensional or symmetrical ones and can be easily measured and evaluated. But asymmetrical temperature fields are more complicated. A sufficient examination of asymmetric temperature fields cannot be done without the use of a tomographic approach. Tomographic approach requires a large number of different projections. In case of DHI it would be necessary to use many digital sensors in the measurement setup to obtain digital holograms for different view in directions. However as was demonstrated in chapter above there is smart way - have only one digital sensor for a periodical coherent phenomenon. Moreover with use of a rotation stage one can capture the area of interest from different directions in precisely defined relative phase of the phenomenon [9].

The experimental principle setup represents Fig. 6 . Object under investigation is SJ. The generator of SJ (cavity and pairs of blowers) is placed on the rotation stage. In the first step the sequence of digital holograms for defined relative delays is captured (whole phenomenon period). One can capture number of holograms for each delay for the subsequent averaging. In the second step the rotation stage is turned at the specific angle and again is captured sequence of holograms. This process is repeated for sufficient number of angles. The interference phase distributions are computed for all holograms. Then is preformed backprojection (12) and all rows of the 2D back-projected field are stecked to get 3D volume data. The final result $3 \mathrm{D}$ volume data of the temperature field are displayed in Fig. 9.
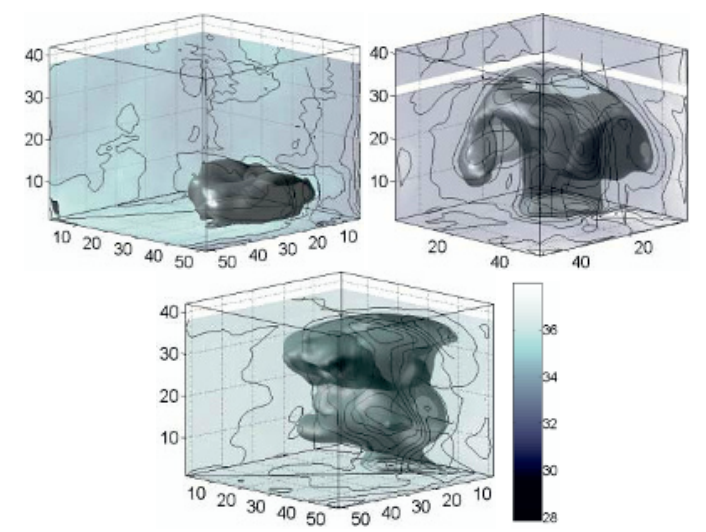

Fig. 9: The 3D volume data of the computed temperature field $\left[{ }^{\circ} \mathrm{C}\right]$ of relative delay time $3,2 \mathrm{~ms}, 9,6 \mathrm{~ms}$ and $19,2 \mathrm{~ms}$.

\section{pros + Gives 3D view of temperature field. Fast periodic (coherent) phenomena measurement. \\ cons - Complex to built up an and adjust. Costly due to need of camera with sync. Long time of acquirement of frames.}

\subsection{Large area measurement}

As was mentioned the size of the measuring area is given by the size of the beamsplitter $\mathrm{BS}_{2}$. For wide area measurement the proper beam expander is inevitable in the setup with double path. Double paths setup brings the benefit of double sensitivity but on the other hand any wavefront distortions caused by imperfections in the optical system of expander are boubled as well. The expander imperfections could origin either from improper assembly of expander or more frequently from non optimized design. The working principle is shown in Fig. 10.

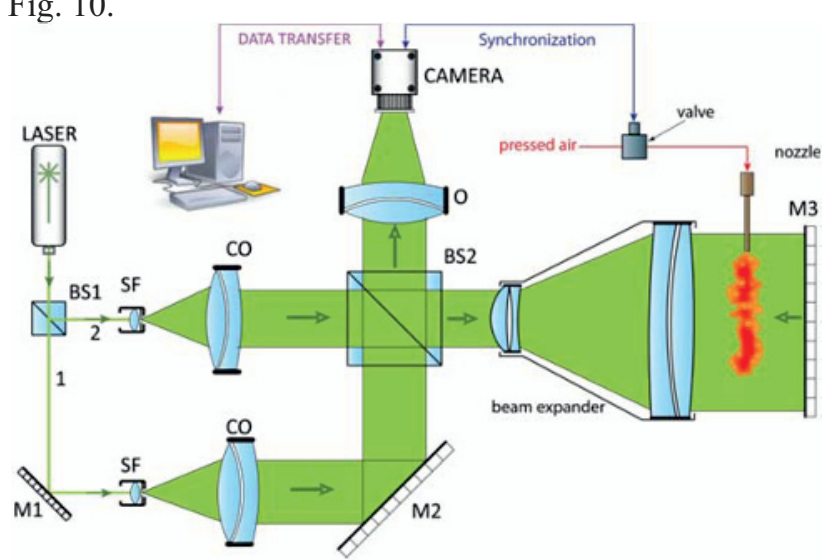

Fig. 10: DHI setup with double path and with beam expander. 
Previously we assembled the expander form off the shelf components but the obtained results were not acceptable. Holograms embody aberration and distortion. This led us to design, optimize and build up expander suitable for our double path setup [10]. There were few requirements and conditions which had to be fulfilled to obtain the BE with satisfactory performance - simplicity, compactness, minimal wavefront aberration and extension (magnification) at least $10 \mathrm{x}$. We modified standard telescope design and by optimization of two doublets we were able to get aplanatic system of the expander. The output diameter of the beam is $120 \mathrm{~mm}$. The function of the beam expander demonstrates experiment of the measurement of the pressed air puff from the weaving nozzle (see Fig. 11). The high speed camera (5000FPS) was used for capturing holograms this fast developing pressure field.

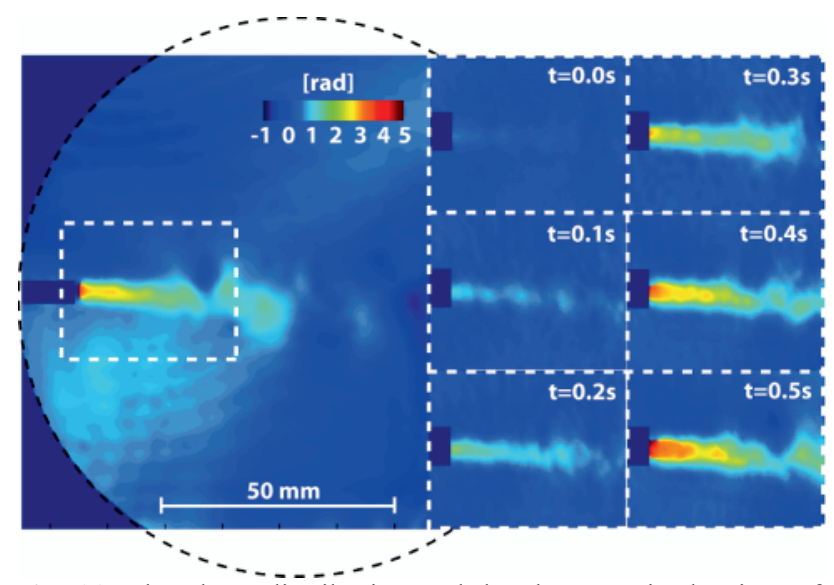

Fig. 11: The phase distribution and development in the time of the pressured air puff.

\footnotetext{
pros + Measurement in large area (only depends on parameters of expander and laser used). Large area plus all above mentioned benefits.

cons - Complicated to built up and very time consuming to adjust.

Costly due to need of on demand designed and manufactured optical components.
}

\section{Sumary}

Few different techniques for refractive index distribution measurement were developed, comprehensively tested and later exploited in research and development done in laboratories of Faculty of mechatronics in Technical University in Liberec. We described those selected techniques and compared its strong and week points. Condensed application properties list was highlighted.

\section{Acknowledgements}

We gratefully acknowledge the support of the GACR (No. P101-11/J019).

\section{References}

1. F. Mayinger, O. Feldmann, Optical Measurements: Techniques and Applications, ISBN 10: 0387567658, Springer-Verlag (July 1994)

2. U. Schnars, W. Jueptner, Digital Holography, ISBN 3-540-21934, Springer 2005.

3. T. Kreis, Handbook of Holographic Interferometry: Optical and Digital Method, Berlin, Wiley, 2004

4. Y.Zhang, G.A.Ruff, Meas. Sci. Technol. 5, 495, (1994)

5. D. W. Sweeney, C. M. Vest, Applied Optics 12, 2649 (1973)

6. V. Lédl, T. Vít, R. Doleček, P. Psota, EPJ Web of Conferences 25, 02014 (2012), DOI: 10.1051/epjconf/20122502014

7. R. Dolecek, P. Psota, V. Lédl, T. Vít, J. Václavík, V. Kopecký, Applied Optics 52 (1), A319-A325 (2013).

8. Z. Trávníček, V. Tesař, Heat Mass Transfer 46, 3291 (2003)

9. R. Dolecek, P. Psota, V. Lédl, T. Vít, J. Václavík, V. Kopecký, Digital Holography and Three-

Dimensional Imaging (2012), DOI: 10.1364/DH.2012.DSu5B.2

10. V. Lédl, P. Psota, T. Vít, R. Doleček, Fringe 2013, 577 (2014). 